\title{
MODELO DE LOCALIZAÇÃO DE FACILIDADES PARA A INSTALAÇÃO DE PÓLOS PARA COLETA DE RESÍDUOS NA REGIÃO DE CURITIBA
}

\author{
Neida M. Patias Volpi, Paulo Henrique Siqueira, \\ Luzia Vidal de Souza, Yuan Jin Yun
}

\author{
Universidade Federal do Paraná \\ neida@ufpr.br, paulohs@ufpr.br, luzia@ufpr.br, yuanjy@gmail.com
}

Resumo: Em função do desafio que é o recolhimento e tratamento do lixo este trabalho propõe a utilização de técnicas matemáticas para encontrar as localizações ótimas para a implantação de estações de tratamento dos resíduos sólidos da Região Metropolitana de Curitiba (RMC), visando reduzir custos de transporte e melhorar o aproveitamento do lixo, com o processamento dos materiais, transformando-os em adubo e fonte energética. Para a realização do trabalho, foram utilizadas as seguintes informações: densidade de lixo por setor da região em estudo; localização de cada possível local para a implantação dos polos de tratamento de lixo; e a distância máxima a ser percorrida pelos caminhões até os polos. Um modelo de programação linear misto é proposto para o problema de coleta de resíduos. $\mathrm{O}$ problema é resolvido em dois estágios denominados de Setorização e Quantidade de Caminhões, respectivamente. No primeiro estágio os locais de instalação dos polos de tratamento são definidos em função dos volumes a serem recolhidos e da frequência de atendimento. No segundo estágio a quantidade de caminhões necessários é calculada. Foram realizadas 5 simulações com diferentes possibilidades de escolhas para instalação de polos de tratamento. O resultado obtido mostra a quantidade de toneladas por polo escolhido, separados por dia da semana e a frota de caminhões necessária para o serviço. O modelo apresentado pode ser usado pelos gestores públicos de acordo com os custos vigentes e políticas adotadas. Diferentes designações foram apresentadas em função da quantidade de polos de tratamento a serem construídos. O modelo permite fazer simulações em função do número de instalações e diferentes custos.

Palavras-Chaves: localização, lixo, programação linear mista

Abstract: Depending on the challenge that is the collection and disposal of garbage this paper proposes the use of mathematical techniques to find the optimal locations of treatment stations of solid waste in the Metropolitan Region of Curitiba (RMC), to reduce transport costs and improve the utilization of waste, with the processing of materials, turning them into compost and energy source. The following information was used to perform work, the bulk density of waste per sector in the region under study; location of each possible site for the establishment of centers of waste treatment; and the maximum distance to be traveled by trucks to the poles. A mixed linear programming model is proposed for the problem of waste collection. The problem is solved in two stages called Sectorization and Trucks Quantity, respectively. In the first stage of installation of local treatment centers are defined in terms of volumes to be collected and the frequency of attendance. In the second stage, the number of trucks needed is calculated. Five simulations with different possible choices for installing poles treatment were performed. The result shows the tonnage sent to each polo, separated by weekday and quantity of trucks needed. The presented model can be used by public managers in accordance with existing policies and cost considered. Different choices were given according to the amount of treatment centers to be built. The model allows simulations based on the number of facilities and different costs.

Keywords: localization, garbage, linear mix programming 


\section{INTRODUÇÃO}

O crescimento da população nos últimos anos e a urbanização acelerada traz à tona um grande problema que é a produção volumosa de lixo nos centros urbanos. Do total de lixo produzido nos centros urbanos, cerca de $45 \%$ é descartado nos aterros sanitários ou lixões a céu aberto. Estes resíduos ocupam um espaço que está sendo reduzido diariamente, com o aumento da população. Calcula-se que em 2050, o lixo sobre a terra deverá atingir um trilhão e 500 bilhões de toneladas. Desta forma, o recolhimento e tratamento do lixo continuam sendo um grande desafio para os gestores públicos dos municípios. O lixo doméstico é recolhido por empresas de limpeza pública e, quando não há a seleção e tratamento corretos, os resíduos são jogados em grandes lixões para efeito de decomposição. Depois de certo período, a fermentação desse lixo gera gás metano e chorume proveniente da decomposição dos dejetos. Quando jogados em aterros sanitários, o lixo sofre uma decomposição controlada e pode servir para a fabricação de adubos naturais.

Assim como já ocorre com o lixo industrial e agrícola, o lixo doméstico tem causado um profundo esgotamento dos aterros sanitários nas principais cidades do mundo. Segundo dados do IBGE, no ano de 2008, cerca de 260 mil toneladas de resíduos eram produzidos no Brasil e pequena parte segue para tratamento adequado, em média cerca de 213 mil toneladas de lixo são jogados diariamente em aterros sanitários ou lixões a céu aberto.

Em função do desafio que é o recolhimento e tratamento do lixo este trabalho propõe a utilização de técnicas matemáticas para encontrar as localizações ótimas para a implantação de estações de tratamento dos resíduos sólidos da Região Metropolitana de Curitiba (RMC), visando reduzir custos de transporte e melhorar o aproveitamento do lixo, com o processamento dos materiais, transformando-os em adubo e fonte energética.

Para a realização do trabalho, foram utilizadas as seguintes informações: densidade de lixo por setor da região em estudo; localização de cada possível local para a implantação dos polos de tratamento de lixo; e a distância máxima a ser percorrida pelos caminhões até os polos.

\section{OBJETIVOS}

Com a realização deste trabalho, pretende-se garantir o desenvolvimento sustentável e inovador, descentralizando o serviço de tratamento dos resíduos, bem como permitir o engajamento social e adequação ambiental. Outros objetivos deste trabalho são a ampliação das oportunidades para o desenvolvimento econômico e sustentabilidade das regiões que receberão os centros de tratamento, além do aumento do aproveitamento dos resíduos sólidos através de sua transformação em energia ou adubo e a minimização dos gastos públicos com o transporte e tratamento dos resíduos.

O objetivo principal deste trabalho é implantar um sistema, utilizando técnicas da Pesquisa Operacional, para indicar as localizações ótimas para a instalação de unidades de tratamento e separação de resíduos sólidos da região de Curitiba, visando a melhoria dos serviços prestados pelos gestores dos resíduos sólidos urbanos, com a redução do custo de transporte e aproveitamento dos resíduos.

\section{REVISÃO DA LITERATURA}

Nesta seção são citados alguns artigos com aplicações similares à proposta deste trabalho, com ênfase em problemas de localização de facilidades. Rosário, Carnieri e Steiner (2002), apresentaram uma metodologia que propõe a distribuição espacial de Unidades de Saúde 24 horas, com o objetivo de reduzir a distância média percorrida pelos usuários até as unidades de saúde. A metodologia utiliza Algoritmos Genéticos, Busca Exaustiva, diagramas de Voronoi e o Algoritmo de Teitz \&Bart. Muller (2007) utiliza o modelo de localização para aplicação ao problema de corte guilhotinado bi-dimensional para o arranjo das peças a serem cortadas, de forma a reduzir as perdas de material. O modelo proposto utiliza p-medianas. 
Bobel e Lopes (2007) utiliza a metodologia de localização de facilidades para alocar equipes de emergência de uma rede de distribuição de energia elétrica com o objetivo de reduzir os custos relacionados à interrupções no sistema elétrico e distribuição das equipes para solucionar o problema. O problema foi resolvido utilizando p-medianas e algoritmos genéticos. A redução de custos girou em torno de $13 \%$ dos valores então considerados. Barcelos, Pizzolato e Lorena (2004), estudaram a problemática da localização de escolas públicas na cidade de Vitória no Espírito Santo. Os autores utilizaram informações obtidas com a utilização do software Arcview. Numa primeira etapa os autores avaliam a localização atual das escolas públicas com o objetivo de identificar as localidades onde ocorre excesso ou escassez de vagas e em seguida propõem uma realocação das escolas utilizando o modelo de p-medianas. Menezes, Diallo e Pizzolato (2011), apresentam um estudo sobre o planejamento da rede escolar no Rio de Janeiro, considerando a previsão do crescimento populacional para os anos de 2015 a 2020. Para a solução do problema utilizando a modelagem matemática e o problema das p-medianas capacitado. Carvalho (2011) propõe uma metodologia de análise da localização de escolas em áreas rurais, com o objetivo de reduzir as distâncias que os estudantes percorrem até suas escolas, diariamente. Esta metodologia é baseada nos conceitos da economia regional e utiliza a teoria da localização para resolver o problema. Utiliza pmedianas para determinar as distâncias de deslocamento dos alunos até suas escolas e utiliza distância euclidiana com o fator de correção. A distância de deslocamento dos alunos até suas escolas, o tempo de deslocamento e o custo do transporte foram considerados na construção de um modelo matemático para a solução do problema ILER (índice de localização de escolas rurais). De acordo com este índice é verificada a viabilidade de instalação de uma nova escola em determinada região.

\section{FORMULAÇÃO MATEMÁTICA}

Nesta seção é apresentado o modelo matemático proposto para o problema de coleta de resíduos. Este modelo é composto de dois estágios denominados de Setorização e Quantidade de Caminhões, respectivamente. No primeiro estágio os locais de instalação dos polos de tratamento são definidos em função dos volumes a serem recolhidos e da frequência de atendimento. No segundo estágio a quantidade de caminhões é avaliada. A região a ser estudada é dividida em vários setores, sendo que o recolhimento do lixo pode ser diário ou em apenas alguns dias da semana, como por exemplo $2^{\mathrm{a}}, 4^{\mathrm{a}}$ e $6^{\mathrm{a}}$.

Na solução do problema são consideradas as seguintes hipóteses:

- o resíduo de cada setor é levado para um único polo de tratamento;

- os locais para instalação de polos de tratamento são definidos a priori;

- os polos de tratamento tem capacidade máxima de atendimento fixa.

Algumas avaliações e considerações são feitas no segundo estágio, a saber:

- o cálculo do número de caminhões;

- um caminhão pode atender mais do que um setor restrito à sua capacidade;

- o caminhão faz a entrega diretamente ao polo de tratamento;

- o custo da coleta é feito após a clusterização.

\section{a. Primeiro Estágio: Setorização}

Neste primeiro estágio consideram-se todos os dados e uma setorização da cidade sendo que se supõe conhecido: os locais de instalação dos polos de tratamento; os dias de coleta definidos por bairro; e a quantidade de resíduos a serem coletados.

Os índices utilizados são $i, j$ e $t$, e representam as seguintes informações:

$i=1, \ldots, m$, setores dos municípios;

$j=1, \ldots, n$, possíveis polos de tratamento de resíduos;

$t=1, \ldots, 7$, dias da semana, $1=$ domingo, $2=$ segunda, $\ldots, 7=$ sábado. 
As constantes utilizadas representam as seguintes informações:

Cappolo: capacidade de polo de tratamento;

$d_{m \times n}$ : matriz de distâncias entre setores e polos;

$R$ : distância máxima percorrida de qualquer setor ao polo de tratamento;

$q_{i \times t}:$ matriz de volumes em toneladas a serem recolhidos no dia $t$ do setor $i$;

$c_{i \times j}$ : custo para transportar uma tonelada de resíduo do setor $i$ até o polo $j$;

custopolo : custo estimado de instalação de cada polo de tratamento;

$p$ : parâmetro que determina o número de polos de tratamento.

As variáveis do problema são:

$x_{i j}=\left\{\begin{array}{l}1 \text { se o lixo do setor i e levado ao polo } j \\ 0 \text { caso contrario }\end{array}\right.$

$y_{j}=\left\{\begin{array}{l}1 \text { se o polo } j \text { é instalado } \\ 0 \text { caso contrario }\end{array}\right.$

O modelo proposto é de programação linear mista, sendo que a função objetivo (1) minimiza o custo total semanal de transporte dos resíduos até o polo de tratamento como também o custo de instalação dos mesmos. A restrição (2) indica que cada setor $i$ é atendido por um único polo $j$. A restrição (3) indica que o atendimento é possível se o polo $j$ é instalado. A restrição (4) indica que $p$ polos serão instalados ( $p$ pode ser variável). A restrição (5) indica a distância máxima $R$ que pode ser percorrida do setor $i$ até o polo $j$. A capacidade diária dos polos é tratamento está representada na restrição (6) e os tipos das variáveis estão indicados na restrição (7). Os pesos na função objetivo $\left(\mathrm{w}_{1} \mathrm{e} \mathrm{w}_{2}\right)$ são ajustados em função da política adotada pelos gestores.

$$
\begin{aligned}
& \text { Minimizar } \mathrm{f}=w_{1} \sum_{t=1}^{7} \sum_{i=1}^{m} \sum_{j=1}^{n} c_{i j} d_{i j} q_{i t} x_{i j}+w_{2} \sum_{j=1}^{n} \text { custopolo }_{j} y_{j} \\
& \sum_{j=1}^{n} x_{i j}=1, i=1, \ldots, m \\
& x_{i j} \leq y_{j}, i=1, \ldots, m, j=1, \ldots, n \\
& \sum_{j=1}^{n} y_{j}=p \\
& \sum_{j=1}^{n} d_{i j} x_{i j} \leq R, i=1, \ldots, m \\
& \sum_{i=1}^{m} q_{i t} x_{i j} \leq \text { Cappolo }_{j} y_{j}, j=1, \ldots, m, t=1, \ldots, 7 \\
& x_{i j} \in\{0,1\}, y_{j} \in\{0,1\}
\end{aligned}
$$

\section{b. Segundo Estágio: Quantidade de Caminhões}

Neste estágio utilizaram-se as etapas descritas a seguir para o cálculo do número necessário de caminhões para o transporte dos resíduos aos polos de tratamento.

Para avaliar o volume de resíduos recebidos em cada polo de tratamento $j$ : $V_{j}=\sum_{i=1}^{m} q_{i} \cdot x_{i j}, j=1, \ldots n$

A avaliação do número de caminhões por polo $j$ em função do tipo de caminhões é feita utilizando-se as seguintes equações:

$$
N c a m 5_{j}=\frac{V_{j}}{5} \text { e } N c a m 9_{j}=\frac{V_{j}}{9},
$$


onde Ncam5 e Ncam 9 são as quantidades de caminhões de capacidade de 5 ton e de 9 ton, respectivamente. Para avaliar o número de total de caminhões de todos os tipos, utilizamos as seguintes equações:

$$
\text { TNcam } 5=\sum_{j=1}^{n} \operatorname{Ncam}_{j} y_{j} \text { e } T N \operatorname{Tcam} 9=\sum_{j=1}^{n} N \operatorname{Nam} 9_{j} y_{j} .
$$

A distância total percorrida pelos caminhões designados será dada pela equação:

$$
D T=\sum_{t=1}^{7} \sum_{i=1}^{m} \sum_{j=1}^{n} d_{i j} x_{i j} \text {. }
$$

\section{RESULTADOS DAS SIMULAÇÕES}

Foram realizadas 5 simulações com diferentes possibilidades de escolhas para instalação de polos de tratamento descritas na Tabela 1.

Tabela 1: Simulações propostas

\begin{tabular}{l|l}
\hline Simulação & Descrição \\
\hline 1 & Escolha livre dos 7 polos \\
2 & Uso dos polos de Almirante Tamandaré, São José dos Pinhais, \\
& Fazenda Rio Grande e da Cidade Industrial de Curitiba \\
3 & Idem a simulação 2 com opção de mais um polo \\
4 & Idem a simulação 2 com opção de mais dois polos \\
5 & Uso de 4 polos livres \\
\hline
\end{tabular}

Os parâmetros utilizados no modelo foram: Cappolo $=850$ ton; $R=56 \mathrm{~km} ; c_{i j}=$ $\mathrm{R} \$ 50,00 /$ ton; custopolo $=80 \times 10^{6}$.

Na figura 1 está representada a região do estudo de caso deste trabalho. No mapa estão representados os sete possíveis locais de instalação de polos de tratamento de resíduos localizados na região metropolitana de Curitiba.

Os polos são denominados Bio 1, situado na cidade de Almirante Tamandaré, Bio 2 em Colombo, Bio 3 na cidade de São José dos Pinhais, Bio 4 na Fazenda Rio Grande, Bio 5 em Curitiba, Energia 1 na Cidade Industrial de Curitiba e Energia 2 também na cidade de Fazenda Rio Grande.

\section{a. SimulaÇão 1}

Na primeira simulação a escolha dos polos de tratamento é livre. O resultado obtido e a quantidade de toneladas por polo, separados por dia da semana está representado na Tabela 2 e a quantidade de caminhões por polo, separados por dia da semana na Tabela 3.

Tabela 2 - Quantidades de toneladas por polo, separadas por dia de semana: Simulação 1

\begin{tabular}{l|lllllll|l}
\hline & SEG & TER & QUA & QUI & SEX & SAB & DOM & $\begin{array}{l}\text { Total } \\
\text { semana }\end{array}$ \\
\hline Bio 1 & 584,29 & 236,97 & 584,29 & 236,97 & 584,29 & 236,97 & 164,1 & $2.627,9$ \\
Bio 2 & 701,77 & 315,72 & 701,77 & 315,72 & 701,77 & 315,72 & 0,00 & $3.052,4$ \\
Bio 3 & 353,97 & 670,92 & 353,97 & 670,92 & 353,97 & 670,92 & 0,00 & $3.074,6$ \\
Bio 4 & 56,20 & 16,52 & 56,20 & 16,52 & 56,20 & 16,52 & 0,00 & 218,15 \\
Bio 5 & 109,51 & 390,40 & 109,51 & 390,40 & 109,51 & 390,40 & 0,00 & $1.499,7$ \\
Energia 1 & 588,28 & 849,47 & 588,28 & 849,47 & 588,28 & 849,47 & 136,9 & $4.450,1$ \\
Energia 2 & 70,07 & 68,33 & 70,07 & 68,33 & 70,07 & 68,33 & 0,00 & 415,19 \\
\hline Total & $2.464,0$ & $2.548,3$ & $2.464,0$ & $2.548,3$ & $2.464,0$ & $2.548,3$ & 301,0 & \\
diário & & & & & & & & \\
\hline
\end{tabular}




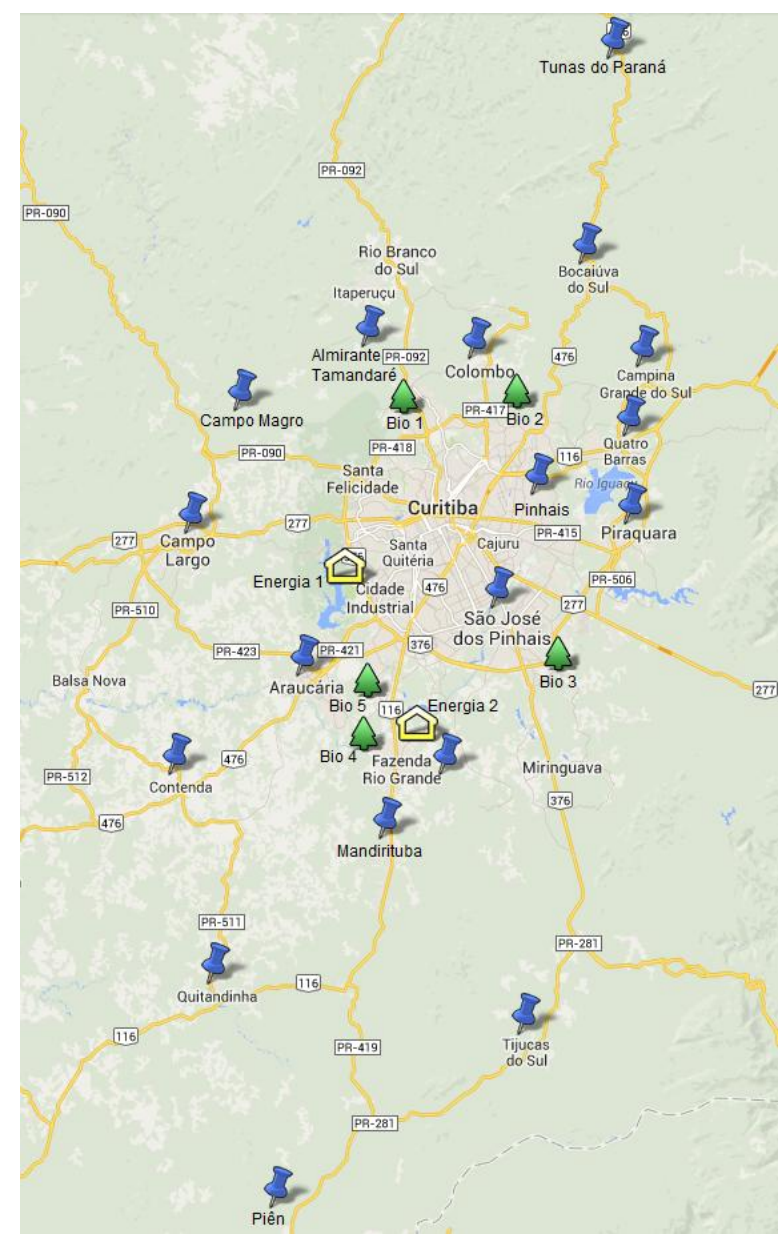

Figura 1 - Mapa com as cidades e os polos de tratamento de resíduos

A Figura 2(a) mostra as designações dos setores separados para cada polo de tratamento, e a quilometragem total encontrada nesta simulação é de $3.176,669 \mathrm{~km}$. A quantidade de caminhões necessária para atender a demanda é 285 com capacidade de 5 toneladas e 197 com capacidade de 9 toneladas.

Tabela 3 - Quantidades de caminhões por polo, separadas por dia de semana: Simulação 1

\begin{tabular}{|c|c|c|c|c|c|c|c|c|c|c|c|c|c|c|c|}
\hline & \multicolumn{2}{|c|}{ SEG } & \multicolumn{2}{|c|}{ TER } & \multicolumn{2}{|c|}{ QUA } & \multicolumn{2}{|c|}{ QUI } & \multicolumn{2}{|c|}{ SEX } & \multicolumn{2}{|c|}{ SAB } & \multicolumn{2}{|c|}{ DOM } & $\begin{array}{l}\text { Total } \\
\text { semanal }\end{array}$ \\
\hline & $9 \mathrm{t}$ & $5 \mathrm{t}$ & $9 \mathrm{t}$ & $5 \mathrm{t}$ & $9 \mathrm{t}$ & $5 \mathrm{t}$ & $9 \mathrm{t}$ & $5 \mathrm{t}$ & $9 \mathrm{t}$ & $5 \mathrm{t}$ & $9 \mathrm{t}$ & $5 \mathrm{t}$ & $9 \mathrm{t}$ & $5 \mathrm{t}$ & $9 \mathrm{t} \quad 5 \mathrm{t}$ \\
\hline$\overline{B i o}$ & 27 & 102 & 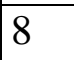 & 46 & 27 & 102 & 8 & 46 & 27 & 102 & , & 46 & 1 & 42 & 106486 \\
\hline Bio & & 63 & 29 & 15 & 56 & 63 & 29 & 15 & 56 & 63 & 29 & 15 & 10 & 0 & $255 \quad 234$ \\
\hline Bio 3 & 32 & 20 & 58 & 50 & 32 & 20 & 58 & 50 & 32 & 20 & 58 & 50 & 0 & 0 & $270 \quad 210$ \\
\hline 4 & 4 & 6 & 1 & 2 & 4 & 6 & 1 & 2 & 4 & 6 & 1 & 2 & 0 & 0 & 24 \\
\hline Bio 5 & 10 & 7 & 33 & 33 & 10 & 7 & 33 & 33 & 10 & 7 & 33 & 33 & 0 & 0 & 129120 \\
\hline er & 38 & 83 & 62 & 99 & 38 & 83 & 62 & 99 & 38 & 83 & 62 & 99 & 1 & 35 & 301581 \\
\hline Ener & 7 & 4 & 6 & 5 & 7 & 4 & 6 & 5 & 7 & 4 & 6 & 5 & 0 & 0 & 27 \\
\hline Total d & 174 & 285 & 197 & 250 & 174 & 285 & 197 & 250 & 174 & 285 & 197 & 250 & 2 & 77 & 2.797 \\
\hline
\end{tabular}

\section{b. SimulaÇão 2}

Na segunda simulação são escolhidos 4 polos de tratamento a priori: Almirante Tamandaré (Bio 1), São José dos Pinhais (Bio 3), Fazenda Rio Grande (Bio 4) e da Cidade Industrial de Curitiba (Energia 1). O resultado obtido e a quantidade de toneladas por polo, separados por dia da semana está representado na Tabela 4 e a quantidade de caminhões por 
polo, separados por dia da semana na Tabela 5.

A Figura 2(b) mostra as designações dos setores separados para cada polo de tratamento, e a quilometragem total encontrada nesta simulação é de $3.593,567 \mathrm{~km}$.

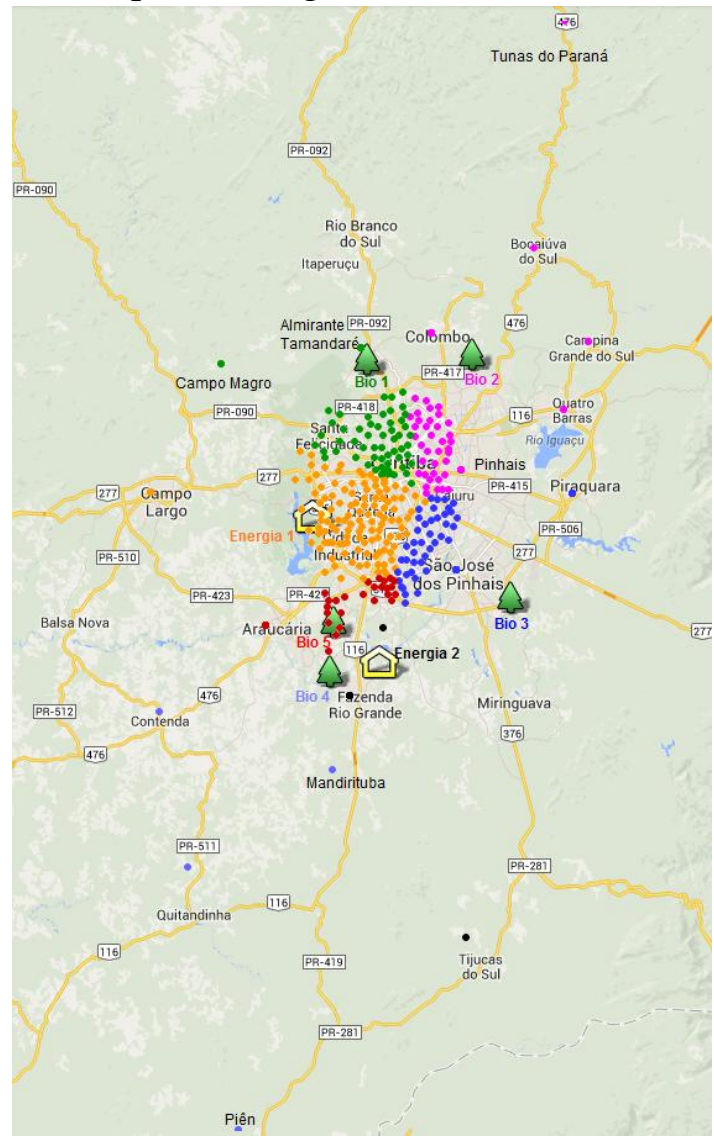

(a)

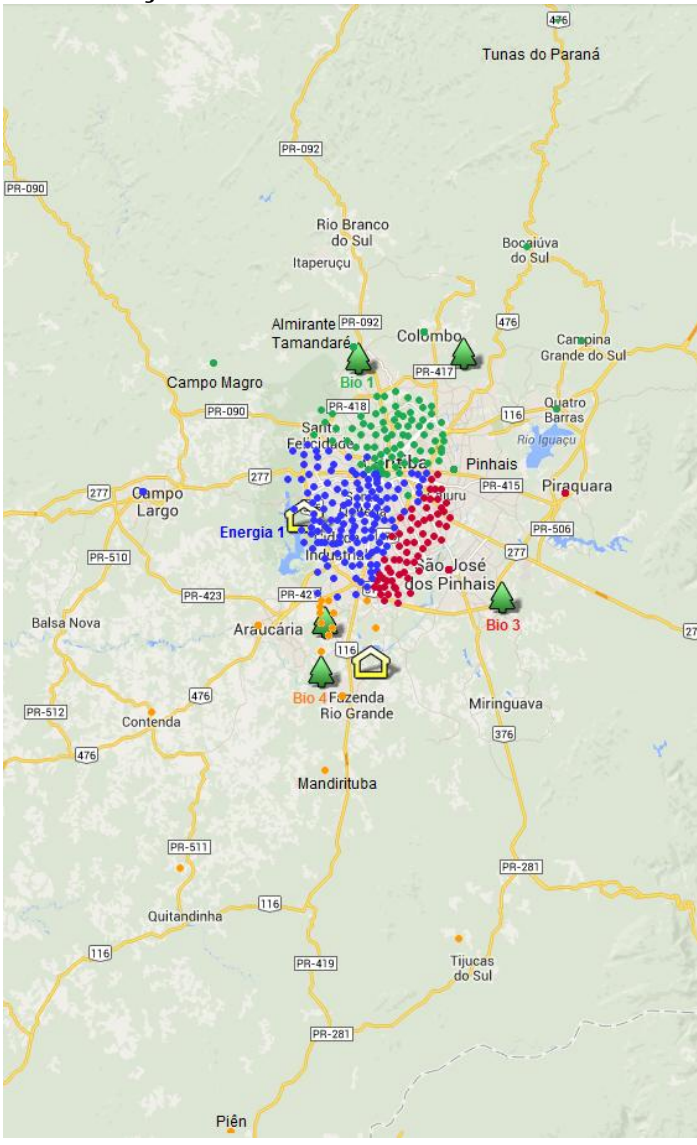

(b)

Figura 2 - Designação dos setores aos polos de tratamento: (a) Simulação 1; (b) Simulação 2

Tabela 4 - Quantidades de toneladas por polo, separadas por dia de semana: Simulação 2

\begin{tabular}{l|lllllll|l}
\hline & SEG & TER & QUA & QUI & SEX & SAB & DOM & $\begin{array}{l}\text { Total } \\
\text { semana } \\
1\end{array}$ \\
\hline Bio 1 & 848,74 & 522,59 & 848,74 & 522,59 & 848,74 & 522,59 & 110,6 & $4.224,6$ \\
Bio 3 & 565,16 & 848,04 & 565,16 & 848,04 & 565,16 & 848,04 & 0,00 & $4.239,6$ \\
Bio 4 & 227,78 & 327,74 & 227,78 & 327,74 & 227,78 & 327,74 & 0,00 & $1.666,5$ \\
Energia 1 & 822,40 & 849,95 & 822,40 & 849,95 & 822,40 & 849,95 & 190,4 & $5.207,4$ \\
\hline $\begin{array}{l}\text { Total } \\
\text { diário }\end{array}$ & $2.464,0$ & $2.548,3$ & $2.464,0$ & $2.548,3$ & $2.464,0$ & $2.548,3$ & 301,0 & \\
\hline
\end{tabular}

Tabela 5 - Quantidades de caminhões por polo, separadas por dia de semana: Simulação 2

\begin{tabular}{|c|c|c|c|c|c|c|c|c|c|c|c|c|c|c|c|}
\hline & \multicolumn{2}{|c|}{ SEG } & \multicolumn{2}{|c|}{ TER } & \multicolumn{2}{|c|}{ QUA } & \multicolumn{2}{|l|}{ QUI } & \multicolumn{2}{|c|}{ SEX } & \multicolumn{2}{|c|}{ SAB } & \multicolumn{2}{|c|}{ DOM } & $\begin{array}{l}\text { Total } \\
\text { semanal }\end{array}$ \\
\hline & $9 \mathrm{t}$ & $5 \mathrm{t}$ & $9 \mathrm{t}$ & $5 \mathrm{t}$ & $9 \mathrm{t}$ & $5 \mathrm{t}$ & $9 t$ & $5 \mathrm{t}$ & $9 \mathrm{t}$ & $5 \mathrm{t}$ & $9 \mathrm{t}$ & $5 \mathrm{t}$ & $9 \mathrm{t}$ & $5 \mathrm{t}$ & $5 \mathrm{t}$ \\
\hline Bio 1 & 51 & 116 & 38 & 50 & 51 & 116 & 38 & 50 & 51 & 116 & 38 & 50 & 0 & 29 & $267 \quad 527$ \\
\hline Bio 3 & 51 & 33 & 72 & 67 & 51 & 33 & 72 & 67 & 51 & 33 & 72 & 67 & 0 & 0 & 369300 \\
\hline Bio 4 & 21 & 15 & 28 & 26 & 21 & 15 & 28 & 26 & 21 & 15 & 28 & 26 & 0 & 0 & $147 \quad 123$ \\
\hline Energia 1 & 51 & 121 & 59 & 107 & 51 & 121 & 59 & 107 & 51 & 121 & 59 & 107 & 2 & 48 & 332732 \\
\hline Total diário & 174 & 285 & 197 & 250 & 174 & 285 & 197 & 250 & 174 & 285 & 197 & 250 & 2 & 77 & 2.797 \\
\hline
\end{tabular}




\section{c. Simulação 3}

Na terceira simulação os 4 polos de tratamento escolhidos a priori são os mesmos da Simulação 2, acrescentando-se a possibilidade de se escolher mais um polo. O polo adicional escolhido através da formulação matemática é o Bio 2, situado em Colombo. $\mathrm{O}$ resultado obtido e a quantidade de toneladas por polo, separados por dia da semana está representado na Tabela 6 e a quantidade de caminhões por polo, separados por dia da semana na Tabela 7.

A Figura 3(a) mostra as designações dos setores separados para cada polo de tratamento, e a quilometragem total encontrada nesta simulação é de $3.282,811 \mathrm{~km}$.

Tabela 6 - Quantidades de toneladas por polo, separadas por dia de semana: Simulação 3

\begin{tabular}{l|lllllll|l}
\hline & SEG & TER & QUA & QUI & SEX & SAB & DOM & $\begin{array}{l}\text { Total } \\
\text { semana } \\
1\end{array}$ \\
\hline Bio 1 & 626,92 & 279,60 & 626,92 & 279,60 & 626,92 & 279,60 & 206,7 & $2.926,3$ \\
Bio 2 & 701,77 & 329,11 & 701,77 & 329,11 & 701,77 & 329,11 & 0,00 & $3.092,6$ \\
Bio 3 & 353,97 & 825,54 & 353,97 & 825,54 & 353,97 & 825,54 & 0,00 & $3.538,5$ \\
Bio 4 & 227,78 & 264,12 & 227,78 & 264,12 & 227,78 & 264,12 & 0,00 & $1.475,7$ \\
Energia 1 & 553,64 & 849,95 & 553,64 & 849,95 & 553,64 & 849,95 & 94,30 & $4.305,0$ \\
\hline Total & $2.464,0$ & $2.548,3$ & $2.464,0$ & $2.548,3$ & $2.464,0$ & $2.548,3$ & 301,0 & \\
diário & & & & & & & & \\
\hline
\end{tabular}

Tabela 7 - Quantidades de caminhões por polo, separadas por dia de semana: Simulação 3

\begin{tabular}{|c|c|c|c|c|c|c|c|c|c|c|c|c|c|c|c|}
\hline & \multicolumn{2}{|l|}{ SEG } & \multicolumn{2}{|c|}{ TER } & \multicolumn{2}{|c|}{ QUA } & \multicolumn{2}{|c|}{ QUI } & \multicolumn{2}{|c|}{ SEX } & \multicolumn{2}{|c|}{ SAB } & \multicolumn{2}{|c|}{ DOM } & $\begin{array}{l}\begin{array}{l}\text { Total } \\
\text { semanal }\end{array} \\
\end{array}$ \\
\hline & $9 \mathrm{t}$ & $5 \mathrm{t}$ & $9 \mathrm{t}$ & $5 \mathrm{t}$ & $9 \mathrm{t}$ & $5 \mathrm{t}$ & $\mathrm{t}$ & $5 \mathrm{t}$ & $9 \mathrm{t}$ & $5 \mathrm{t}$ & $9 \mathrm{t}$ & $5 \mathrm{t}$ & $9 \mathrm{t}$ & $5 \mathrm{t}$ & $5 \mathrm{t}$ \\
\hline & & & 8 & 58 & 27 & & & & & & o & 8 & & 4 & $06 \quad 570$ \\
\hline & & 63 & 30 & 16 & 56 & 63 & 30 & 16 & 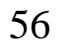 & 63 & 30 & 16 & 0 & 0 & $258 \quad 237$ \\
\hline & & 20 & 70 & 65 & 32 & 20 & 76 & 65 & & 20 & 70 & 65 & & & $306 \quad 255$ \\
\hline & & 15 & 23 & 20 & 21 & 15 & 2. & 20 & & 15 & & 20 & & & 132105 \\
\hline & & 73 & 66 & 91 & 38 & 73 & 66 & 91 & 30 & 73 & 66 & 91 & 1 & 23 & $313 \quad 515$ \\
\hline Total diário & 174 & 285 & 197 & 250 & 174 & 285 & 197 & 250 & 174 & 285 & 197 & 250 & 2 & 77 & 2.797 \\
\hline
\end{tabular}

\section{d. Simulação 4}

$\mathrm{Na}$ quarta simulação os 4 polos de tratamento escolhidos a priori são os mesmos da Simulação 2, acrescentado a possibilidade de escolher mais dois locais para tratamento. Os polos adicionais encontrados pela formulação matemática foram o Bio 2 e o Bio 5 . $\mathrm{O}$ resultado obtido e a quantidade de toneladas por polo, separados por dia da semana está representado na Tabela 8 e a quantidade de caminhões por polo, separados por dia da semana na Tabela 9.

Tabela 8 - Quantidades de toneladas por polo, separadas por dia de semana: Simulação 4

\begin{tabular}{l|lllllll|l}
\hline & SEG & TER & QUA & QUI & SEX & SAB & DOM & $\begin{array}{l}\text { Total } \\
\text { semana }\end{array}$ \\
\hline Bio 1 & 584,29 & 236,97 & 584,29 & 236,97 & 584,29 & 236,97 & 164,1 & $2.627,9$ \\
Bio 2 & 701,77 & 315,72 & 701,77 & 315,72 & 701,77 & 315,72 & 0,00 & $3.052,4$ \\
Bio 3 & 353,97 & 670,92 & 353,97 & 670,92 & 353,97 & 670,92 & 0,00 & $3.074,6$ \\
Bio 4 & 126,27 & 75,98 & 126,27 & 75,98 & 126,27 & 75,98 & 0,00 & 606,73 \\
Bio 5 & 109,51 & 399,27 & 109,51 & 399,27 & 109,51 & 399,27 & 0,00 & $1.526,3$ \\
Energia 1 & 588,28 & 849,47 & 588,28 & 849,47 & 588,28 & 849,47 & 136,9 & $4.450,1$ \\
\hline Total dia & $2.464,0$ & $2.548,3$ & $2.464,0$ & $2.548,3$ & $2.464,0$ & $2.548,3$ & 301,0 & \\
\hline
\end{tabular}


Tabela 9 - Quantidades de caminhões por polo, separadas por dia de semana: Simulação 4

\begin{tabular}{|c|c|c|c|c|c|c|c|c|c|c|c|c|c|c|c|c|}
\hline & \multicolumn{2}{|c|}{ SEG } & \multicolumn{2}{|c|}{ TER } & \multicolumn{2}{|c|}{ QUA } & \multicolumn{2}{|c|}{ QUI } & \multicolumn{2}{|c|}{ SEX } & \multicolumn{2}{|c|}{ SAB } & \multicolumn{2}{|c|}{ DOM } & \multicolumn{2}{|c|}{$\begin{array}{l}\text { Total } \\
\text { semanal } \\
\end{array}$} \\
\hline & $9 \mathrm{t}$ & $5 \mathrm{t}$ & $9 \mathrm{t}$ & $5 \mathrm{t}$ & $9 \mathrm{t}$ & $5 \mathrm{t}$ & $9 \mathrm{t}$ & $5 \mathrm{t}$ & $9 \mathrm{t}$ & $5 \mathrm{t}$ & $9 \mathrm{t}$ & $5 \mathrm{t}$ & $9 \mathrm{t}$ & $5 \mathrm{t}$ & $9 \mathrm{t}$ & $5 \mathrm{t}$ \\
\hline$\overline{\mathrm{Bio}}$ & 27 & 102 & 8 & 46 & 27 & 102 & 8 & 46 & 27 & 102 & 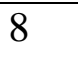 & 46 & 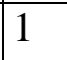 & 42 & 27 & 102 \\
\hline Bio & 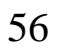 & 63 & 29 & 15 & 56 & 63 & 29 & 15 & 50 & 63 & 29 & 15 & J & U & 56 & 63 \\
\hline Bio & 32 & 20 & 58 & 50 & 32 & 20 & 58 & 50 & 32 & 20 & 58 & 50 & 0 & 0 & 32 & 20 \\
\hline Bio & 11 & 10 & 7 & 5 & 11 & 10 & 7 & 5 & 11 & 10 & | & 5 & 0 & 0 & 11 & 10 \\
\hline & 10 & 7 & 33 & 35 & 10 & 7 & 33 & 35 & 10 & 7 & 33 & 35 & 0 & 0 & 10 & 7 \\
\hline Energi & 38 & 83 & 62 & 99 & 38 & 83 & 62 & 99 & 38 & 83 & 62 & 99 & 1 & 35 & 38 & 83 \\
\hline Total diário & 174 & 285 & 197 & 250 & 174 & 285 & 197 & 250 & 174 & 285 & 197 & 250 & 2 & 77 & 2.79 & \\
\hline
\end{tabular}

A Figura 3(b) mostra as designações dos setores separados para cada polo de tratamento, e a quilometragem total encontrada nesta simulação é de $3.186,347 \mathrm{~km}$.

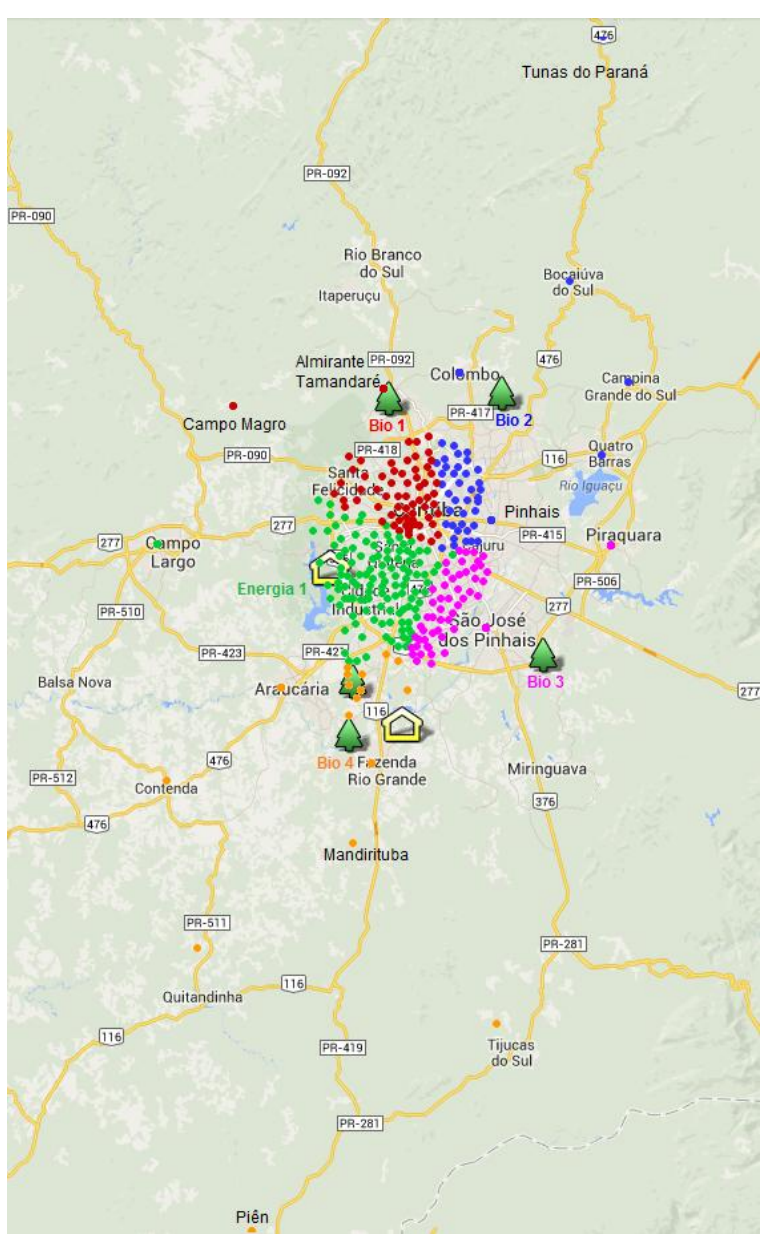

(a)

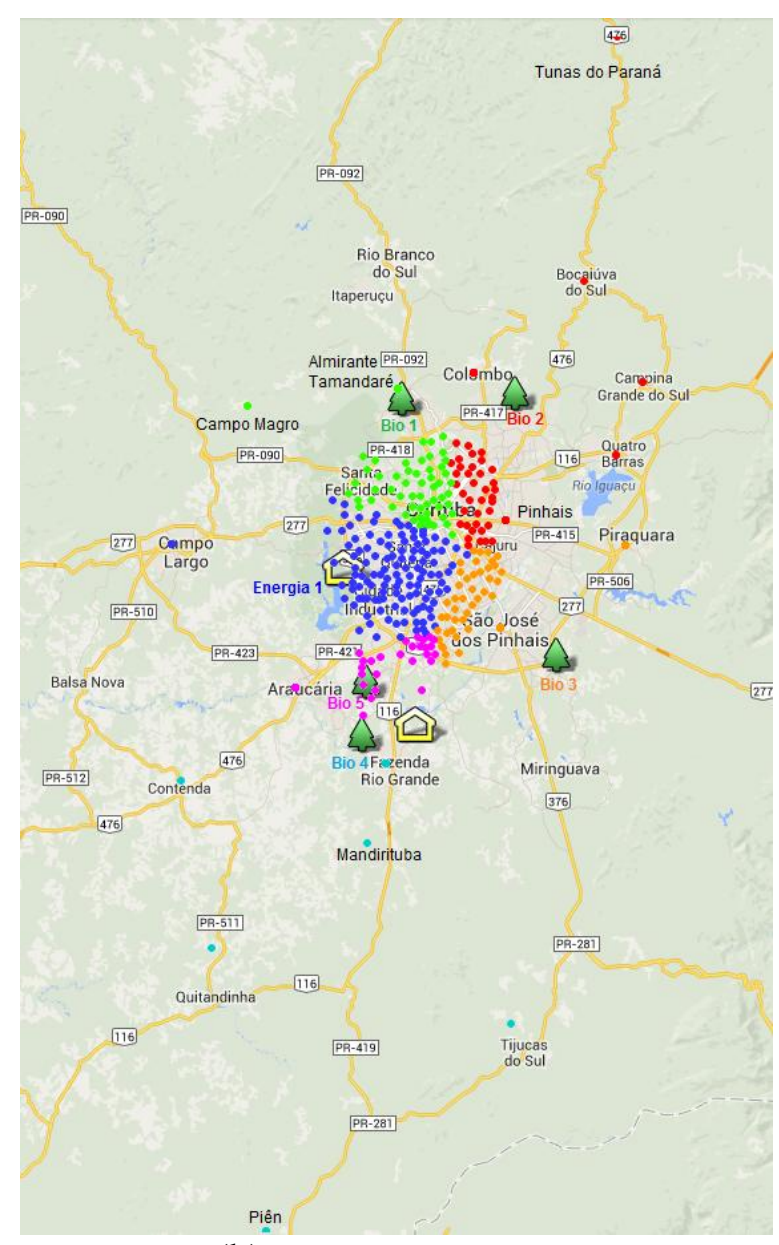

(b)

Figura 3 - Designação dos setores aos polos de tratamento: (a) Simulação 3; (b) Simulação 4

\section{e. Simulação 5}

Na quinta e última simulação foram escolhidos livremente 4 polos de tratamento. Os polos escolhidos através da formulação matemática foram o Bio 1, Bio 3, Bio 5 e Energia 1. O resultado obtido e a quantidade de toneladas por polo, separados por dia da semana está representado na Tabela 10 e a quantidade de caminhões por polo, separados por dia da 
semana na Tabela 11.

Tabela 10 - Quantidades de toneladas por polo, separadas por dia de semana: Simulação 5

\begin{tabular}{l|lllllll|l}
\hline & SEG & TER & QUA & QUI & SEX & SAB & DOM & $\begin{array}{l}\text { Total } \\
\text { semana }\end{array}$ \\
\hline Bio 1 & 848,93 & 467,15 & 848,93 & 467,15 & 848,93 & 467,15 & 78,55 & $4.026,7$ \\
Bio 3 & 543,35 & 697,82 & 543,35 & 697,82 & 543,35 & 697,82 & 0,00 & $3.723,4$ \\
Bio 5 & 225,16 & 534,52 & 225,16 & 534,52 & 225,16 & 534,52 & 0,00 & $2.279,0$ \\
Energia 1 & 846,65 & 848,84 & 846,65 & 848,84 & 846,65 & 848,84 & 222,5 & $5.308,9$ \\
\hline $\begin{array}{l}\text { Total } \\
\text { diário }\end{array}$ & $2.464,0$ & $2.548,3$ & $2.464,0$ & $2.548,3$ & $2.464,0$ & $2.548,3$ & 301,0 & \\
\hline
\end{tabular}

Tabela 11 - Quantidades de caminhões por polo, separadas por dia de semana: Simulação 5

\begin{tabular}{|c|c|c|c|c|c|c|c|c|c|c|c|c|c|c|}
\hline & \multicolumn{2}{|c|}{ SEG } & \multicolumn{2}{|c|}{ TER } & \multicolumn{2}{|c|}{ QUA } & \multicolumn{2}{|c|}{ QUI } & \multicolumn{2}{|l|}{ SEX } & \multicolumn{2}{|c|}{ SAB } & DOM & $\begin{array}{l}\text { Total } \\
\text { semanal }\end{array}$ \\
\hline & $9 \mathrm{t}$ & $5 \mathrm{t}$ & $9 \mathrm{t}$ & $5 \mathrm{t}$ & $9 \mathrm{t}$ & $5 \mathrm{t}$ & $9 t$ & $5 \mathrm{t}$ & $9 \mathrm{t}$ & $5 \mathrm{t}$ & $9 \mathrm{t}$ & $5 \mathrm{t}$ & $9 \mathrm{t} 5 \mathrm{t}$ & $9 \mathrm{t} \quad 5 \mathrm{t}$ \\
\hline$\overline{\text { Bio } 1}$ & 58 & 103 & 36 & 39 & 58 & 103 & 36 & 39 & 58 & 103 & 36 & 39 & 20 & 282446 \\
\hline Bio 3 & 49 & 32 & 60 & 53 & 49 & 32 & 60 & 53 & 49 & 32 & 60 & 53 & 0 & $327 \quad 255$ \\
\hline Bio 5 & 20 & 16 & 45 & 46 & 20 & 16 & 45 & 46 & 20 & 16 & 45 & 46 & 0 & 195186 \\
\hline Energia 1 & 47 & 134 & 56 & 112 & 47 & 134 & 56 & 112 & 47 & 134 & 56 & 112 & 57 & 311795 \\
\hline Total diário & 174 & 285 & 197 & 250 & 174 & 285 & 197 & 250 & 174 & 285 & 197 & 250 & 77 & 2.797 \\
\hline
\end{tabular}

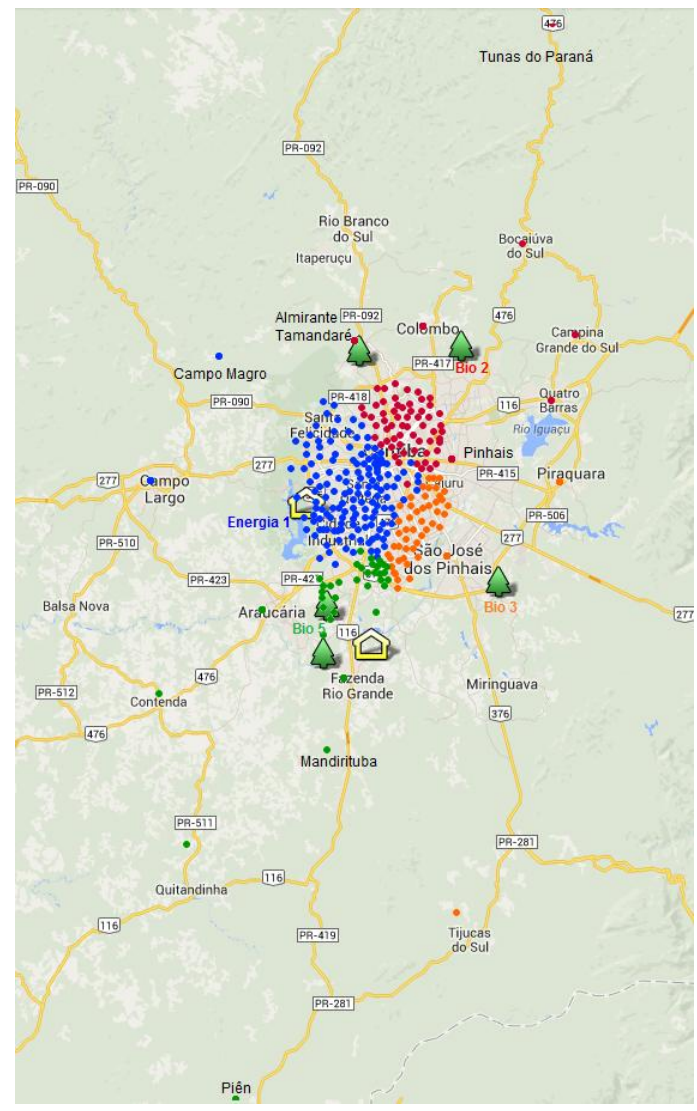

Figura 4 - Designação dos setores aos polos de tratamento: Simulação 5 
A Figura 4 mostra as designações dos setores separados para cada polo de tratamento, e a quilometragem total encontrada nesta simulação é de $3.499,329 \mathrm{~km}$.

\section{CONCLUSÕES}

De acordo com os resultados das simulações apresentadas pode-se concluir que a melhor alternativa seria da Simulação 1, com a construção dos 7 polos de tratamento e a quilometragem total de 3.176,669 $\mathrm{km}$ diários com o transporte de resíduos aos polos de tratamento. A melhor opção para a construção de apenas 4 polos de tratamento é apresentada na simulação 5, com quilometragem total de 3.499,329 km semanais de transporte de resíduos aos polos de tratamento. O modelo apresentado pode ser usado pelos gestores públicos de acordo com os custos vigentes e políticas adotadas. Diferentes designações foram apresentadas em função da quantidade de polos de tratamento a serem construídos. O modelo permite fazer simulações em função do número de instalações e ao mesmo tempo avaliar o número de caminhões necessários para fazer a entrega dos resíduos.

\section{REFERÊNCIAS BIBLIOGRÁFICAS}

[1] BARCELOS, F. B.; PIZZOLATO, N. D.; LORENA, L. A. Localização de escolas do ensino fundamental com modelos capacitados e não capacitados: caso de Vitória/ES. Pesquisa Operacional, v. 24, p 133-149, 2004.

[2] CARVALHO, L. C. Metodologia de análise para a localização de escolas em áreas rurais. Tese de doutorado. Departamento de Eng. Civil e ambiental. Programa de Pós Graduação em Transporte. Universidade de Brasília. (2011)

[3] MENEZES, R.; DIALLO, M.; PIZZOLATO, N. D. Escolas públicas em Guaratiba, Rio de Janeiro: aplicação do modelo de p-medianas capacitado e de máxima cobertura. XLII SBPO, 2011.

[4] MULLER, A. T. G. I. Aplicação de p-medianas ao problema do corte guilhotinado bidimensional para peças regulares. XVI Seminário de Computação: FURB, 2007.

[5] NDIAVE, M.; ALFARES, H. Modelling health care facility location for moving population groups. Systems Engineering Departament, King Fahd University of Petroleum and Minerals, Dhahran 31261, Saudi Arabia. Computers \&Operations Research 35, p. 2154 - 2161, 2008.

[6] ROSÁRIO, R. R. L.; CARNIERI, C.; STEINER, M. T. A proposta de solução para o problema das p-medianas na localização de unidades de saúde 24 horas. XXII ENEGEP, 2002. 